\title{
POLYMORPHISM OF EXTRACELLULAR SUPEROXIDE DISMUTASE (EC-SOD) GENE: RELATION TO THE MUTATION RESPONSIBLE FOR HIGH EC-SOD LEVEL IN SERUM
}

\author{
Harutaka YamadA, ${ }^{1, *}$ Yasukazu Yamada, ${ }^{2}$ Tetsuo AdACHI, ${ }^{3}$ \\ Haruko Goto, ${ }^{2}$ Nobuaki OGasawara, ${ }^{2}$ Arao FutenMa, ${ }^{1}$ \\ Mitsuru Kitano, ${ }^{1}$ Hironobu Mryal, ${ }^{1}{ }^{1}$ Atsushi Fukatsu, ${ }^{1}$ \\ Kazuyuki HIRANO, ${ }^{4}$ and Shinichi KaKUMU ${ }^{1}$ \\ ${ }^{1}$ First Department of Internal Medicine, Aichi Medical University, \\ Nagakute, Aichi 480-11, Japan \\ ${ }^{2}$ Department of Genetics, Institute for Developmental Research, \\ Aichi Human Service Center, Kasugai, Aichi 480-03, Japan \\ ${ }^{3}$ Laboratory of clinical Pharmaceutics, Gifu Pharmaceutical University, \\ Gifu 502, Japan \\ ${ }^{4}$ Department of Pharmaceutics, Gifu Pharmaceutical University, \\ Gifu 502, Japan
}

Summary Extracellular superoxide dismutase (EC-SOD) with amino acid substitution R213G generated by the nucleotide substitution $760 \mathrm{C} \rightarrow$ $G$ in the heparin binding domain is responsible for the high EC-SOD level in serum. We identified the two DNA polymorphic sites in the coding region of EC-SOD gene related to the $760 \mathrm{C} \rightarrow \mathrm{G}$ and determined the allele frequencies. The polymorphisms were $\mathrm{A}$ and $\mathrm{G}$ at nucleotide position (nt.) 241 and $\mathrm{C}$ and $\mathrm{T}$ at nt. 280 near the $\mathrm{N}$-terminal. The haplotype frequencies in Japanese were 241 A280C: 0.45, 241G280T: 0.37, and 241G280C: 0.18 . The haplotype of 241A280T did not exist. The mutation $760 \mathrm{C} \rightarrow \mathrm{G}$ must occur on the allele having the haplotype of 241G280T.

Key Words extracellular superoxide dismutase (EC-SOD), DNA polymorphism, haplotype, mutation

\section{Introduction}

Extracellular superoxide dismutase (EC-SOD) is an SOD isozyme mainly distributed in the extracellular fluid. It is located on the endothelial cell surface

Received January 21, 1997; Accepted March 5, 1997.

* To whom correspondence should be addressed. 
according to the heparin binding capacity. The EC-SOD gene is located on human chromosome 4 (Gallagher et al., 1992). Its cDNA sequence was reported by Hjalmarsson et al. (1987), and the entire coding region was included in the same exon (Yamada et al., 1995). Recently, a single point mutation of the $\mathrm{C}$ to $\mathrm{G}$ (760C $\rightarrow G$ ) near the carboxyl terminal has been identified in $2 \%$ of the plasma donors in Sweden (Sandstöm et al., 1994), in 3.3\% in USA (Folz et al., 1994), and 6\% in Japan (Yamada et al., 1995). The amino acid substitution (R213G) is important for the heparin binding capacity which is one of the characteristics of EC-SOD (Adachi et al., 1996). In the process of the gene analysis, we found two polymorphic sites near the N-terminal of EC-SOD. Herein, we determined the frequencies of these polymorphisms, and clarified their relationship with the mutation $760 \mathrm{C} \rightarrow$ $G$ responsible for the high EC-SOD level in serum.

\section{Materials and Methods}

All the methods of EC-SOD gene analysis were described previously (Yamada et al., 1995). DNA fragments (910 bp) including the entire coding region of EC-SOD gene were amplified using a primers pair (SE1A and SE1B) from genomic DNAs. The polymerase chain reaction (PCR) was carried out for 40 cycles $\left(94^{\circ} \mathrm{C}, 1 \mathrm{~min} ; 60^{\circ} \mathrm{C}, 1 \mathrm{~min} ; 70^{\circ} \mathrm{C}, 2 \mathrm{~min}\right)$. After electrophoresis in a low melting point agarose gel (1.5\%) (Ultra Pure, BRL, Gaithersburg, USA), the fragments were extracted from the gels, purified by PEG precipitation, and the nucleotide sequences were determined according to the simplified direct sequencing method using the 7-deaza-dGTP Reagent Kit for Sequenase ver. 2 (Amersham USB, Little Chalfont, England). The mutation site $(760 \mathrm{C} \rightarrow \mathrm{G})$ were sequenced using a primer SE2B, and two polymorphic sites using SE5B. For the cloning study, PCR products was ligated to the PCR II vecter (Invitrogen, San Diego, USA), separated to the single clone and the sequence checked.

\section{Results and Discussion}

From the genomic DNAs derived from 253 Japanese healthy persons and hemodialysis patients, the coding region of EC-SOD was PCR-amplified using the specific primers. The substitutions of the $A$ to $G$ at nt. $241(241 \mathrm{~A} \rightarrow G)$, and the $C$ to $\mathrm{T}$ at $\mathrm{nt} .280(280 \mathrm{C} \rightarrow \mathrm{T})$ compared with the sequences described by Hjalmarsson et al. (1987), were detected by the direct sequencing of the PCR products. The substitution $241 \mathrm{~A} \rightarrow \mathrm{G}$ generates the amino acid change of Thr to Ala (T40A), but $280 \mathrm{C} \rightarrow \mathrm{T}$ is silent. The substitution $\mathrm{T} 40 \mathrm{~A}$ did not affect heparin binding capacity or the specific activity of EC-SOD (unpublished data). The $241 \mathrm{G}$ creates a new Bss HII restriction site.

The subjects were classified into; 222 without the mutation $760 \mathrm{C} \rightarrow \mathrm{G}$, and 29 heterozygous, and 4 homozygous for the mutation. As shown in Table 1, there were differences in frequencies of the two polymorphisms between the cases with and without the mutation $760 \mathrm{C} \rightarrow \mathrm{G}$. The frequencies of $241 \mathrm{G}$ and $280 \mathrm{~T}$ in the 
Table 1. The allele frequencies of the DNA polymorphisms in the groups with and without the mutation $760 \mathrm{C} \rightarrow \mathrm{G}$.

\begin{tabular}{cccc}
\hline & Without $760 \mathrm{C} \rightarrow \mathrm{G}$ & \multicolumn{2}{c}{ With $760 \mathrm{C} \rightarrow \mathrm{G}$} \\
\cline { 3 - 4 } $\begin{array}{c}\text { Allele } \\
(\mathrm{n})\end{array}$ & & Heterozygote & Homozygote \\
\hline $241 \mathrm{~A}$ & 444 & 58 & 8 \\
$241 \mathrm{G}$ & 0.45 & 0.26 & 0.00 \\
$280 \mathrm{C}$ & 0.55 & 0.74 & 1.00 \\
$280 \mathrm{~T}$ & 0.63 & 0.39 & 0.00 \\
\hline
\end{tabular}

Table 2. The haplotype frequencies of the DNA polymorphisms in the individuals without the mutation.

\begin{tabular}{lcc}
\hline Haplotype & \multicolumn{3}{c}{ Frequency } \\
\hline 241A280C & Japanese & Caucasian \\
241 A280T & 0.45 & 0.30 \\
$241 \mathrm{G} 280 \mathrm{C}$ & 0.00 & 0.00 \\
$241 \mathrm{G} 280 \mathrm{~T}$ & 0.18 & 0.55 \\
\hline
\end{tabular}

individuals with the mutation were significantly higher than those without the mutation. In the 4 homozygous individuals, only a haplotype 241G280T was found. These findings suggested that the mutation $760 \mathrm{C} \rightarrow \mathrm{G}$ was closely related to the detected polymorphisms. Furthermore, the substitution 280T may have occurred on the allele with $241 \mathrm{G}$, since all individuals with $280 \mathrm{~T}$ had $241 \mathrm{G}$.

To clarify the relationship between the $760 \mathrm{C} \rightarrow \mathrm{G}$ and haplotypes of two polymorphic sites, we carried out cloning studies of the heterozygous individuals for both polymorphisms (241A and $\mathrm{G}, 280 \mathrm{C}$ and $\mathrm{T}$ ). We analyzed the first 20 clones derived from 5 individuals with the mutation $760 \mathrm{C} \rightarrow \mathrm{G}$ on one allele. All 10 clones with the mutation were a haplotype of $241 \mathrm{G} 280 \mathrm{~T}$, whereas the other 10 clones without the mutation were a haplotype of 241 A280C. Analyses of 40 clones derived from 10 individuals without the $760 \mathrm{C} \rightarrow \mathrm{G}$ showed the following results: 10 clones with a haplotype of $241 \mathrm{~A} 280 \mathrm{C}, 8$ clones with $241 \mathrm{G} 280 \mathrm{C}$, and 22 clones with $241 \mathrm{G} 280 \mathrm{~T}$. No clone with a haplotype of $241 \mathrm{~A} 280 \mathrm{~T}$ was detected in any clone with or without the $760 \mathrm{C} \rightarrow \mathrm{G}$. These findings suggested the absence of an allele having the haplotype of 241A280T and the occurrence of a mutation $760 \mathrm{C} \rightarrow \mathrm{G}$ associated with a high EC-SOD level in serum on the allele of the haplotype of 241G280T.

The frequencies of the haplotypes in the Japanese were calculated from the data in Table 1, based on the absence of 241 A180T (Table 2). The frequency in the Caucasians was also calculated from our preliminary analysis on 10 individuals without the $760 \mathrm{C} \rightarrow \mathrm{G}$. In the frequency of haplotype of $241 \mathrm{G} 280 \mathrm{~T}$ on which the 
$760 \mathrm{C} \rightarrow \mathrm{G}$ seemed to have occurred, the value in the Caucasians $(0.15)$ was significantly lower than that in the Japanese (0.37). This difference may reflect the higher incidence of the high EC-SOD levels in serum in the Japanese due to the mutation $760 \mathrm{C} \rightarrow \mathrm{G}$ which may have occurred on the allele of haplotype of $241 \mathrm{G} 280 \mathrm{~T}$.

\section{REFERENCES}

Adachi T, Yamada H, Yamada Y, Morihara N, Yamazaki N, Murakami T, Futenma A, Kato K, Hirano K (1996): Substitution of glycine for arginine-213 in extracellular-superoxide dismutase impairs affinity for heparine and endothelial cell surface. Biochem J 313: 235-239

Folz RJ, Green LP, Crapo JD (1994): Identification of missense mutation (Arg to Gly) in the critical binding region of the human gene (SOD3) and its association with dramatically increased serum enzyme levels. Hum Mol Genet 3: 2251-2254

Gallagher DS Jr, Gibbs LS, Shatter JB, Womak JE (1992): Somatic cell mapping of bovine EC-SOD and SOD $1 \mathrm{~L}$ loci. Genomics 12: 610-612

Hjalmarsson K, Marklund SL, Engström A, Edulund T (1987): Isolation and sequence of complementary DNA encoding human extracellular superoxide dismutase. Proc Natl Acad Sci USA 84: 6340-6344

Sandström J, Nilsson P, Karlsson K, Marklund SL (1994): 10-fold increase in human plasma extracellular superoxide dismutase content caused by a mutation in heparin-binding domain. J Biol Chem 269: 19163-19166

Yamada H, Yamada Y, Adachi T, Goto H, Ogasawara N, Futenma A, Kitano M, Hirano K, Kato K (1995): Molecular analysis of extracellular superoxide dismutase gene associated with high level in serum. Jpn J Human Genet 40: 177-184 